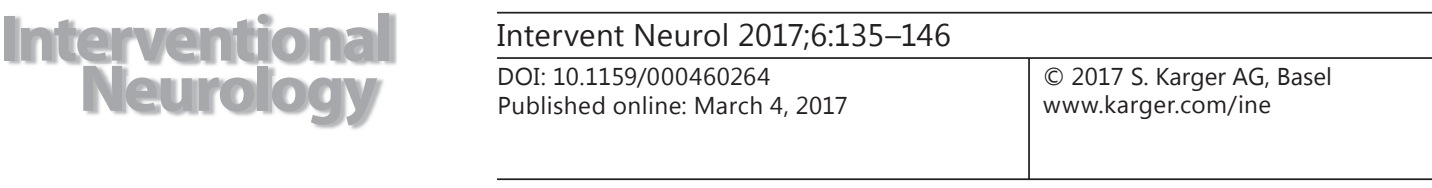

\title{
Rescue Treatment with Pipeline Embolization for Postsurgical Clipping Recurrences of Anterior Communicating Artery Region Aneurysms
}

\author{
Li-Mei Lin $^{a} \quad$ Rajiv R. Iyer ${ }^{b} \quad$ Matthew T. Bender ${ }^{b}$ Thomas Monarch ${ }^{b}$ \\ Geoffrey P. Colby ${ }^{b}$ Judy Huang ${ }^{\text {b }}$ Rafael J. Tamargo ${ }^{b}$ \\ Alexander L. Coon ${ }^{b}$ \\ ${ }^{a}$ Department of Neurosurgery, University of California, Irvine School of Medicine, UC Irvine \\ Medical Center, Orange, CA, and bepartment of Neurosurgery, Johns Hopkins University \\ School of Medicine, The Johns Hopkins Hospital, Baltimore, MD, USA
}

\section{Keywords}

Aneurysm - Endovascular treatment · Flow diversion - Distal access - Pipeline embolization device

\begin{abstract}
Background: Postsurgical clipping aneurysm recurrences or residuals can be difficult to manage with either traditional open microsurgical approaches or endosaccular coiling. Endoluminal parent vessel reconstruction with flow diversion may be an ideal method for treating these recurrences by avoiding reoperative surgery or intraprocedural aneurysm rupture with aneurysm access. Method: We retrospectively reviewed a single-center aneurysm database identifying all anterior communicating artery (ACom) region aneurysms with recurrences after microsurgical clipping. Cases subsequently treated with Pipeline embolization device (PED) were identified for analysis. Results: Nine PED neurointerventions were performed for the treatment of 6 ACom region recurrent aneurysms after surgical clipping (ACom, $n=4$ and A1-A2 junction, $n=2$ ). Of the 6 aneurysms treated, 4 were previously ruptured. Mean patient age was $59.5 \pm 6.9$ years (range 50-67 years). Mean aneurysm size was $5.1 \pm 2.2 \mathrm{~mm}$ (range 3-9 mm). Mean fluoroscopy time was $44.1 \pm 12.4 \mathrm{~min}$. A single PED, deployed from ipsilateral $A 2$ to ipsilateral $A 1$, was used in 6 cases. No instances of periprocedural complications were encountered. Angiographic follow-up was available in all aneurysms; 5 of these 6 (83\%) demonstrated complete aneurysm occlusion. Conclusion: Flow diversion with PED can be a safe and efficacious treatment approach for recurrent ACom region aneurysms after surgical clipping.




\section{Introduction}

Despite the advanced techniques of the modern neuroendovascular era, some centers continue to treat anterior circulation aneurysms beyond the carotid termination, such as anterior communicating artery (ACom) and middle cerebral artery aneurysms with microsurgical clipping [1,2]. Surgical clipping has known risks of residual aneurysm detected on postoperative angiography estimated at approximately $4-8 \%$ and a recurrence rate of $1-2 \%$ [3-5]. Aneurysm residuum and recurrences are associated with risk of growth and risk of rupture. These residuals or recurrences have been managed with reoperative microsurgical clipping; however, second surgeries are frequently challenged with adhesions and fibrosis resulting in higher rates of complications.

Endovascular coiling for treatment of aneurysm residuum or recurrences after surgical clipping has been described as an alternative strategy to surgical reintervention [6-8]. These experiences report the safety and effectiveness of coiling aneurysm remnants in various locations, including the ACom region. However, the traditional endosaccular approach with coil embolization is associated with known risks of recurrence and intraprocedural aneurysm rupture [9-11].

Compared to the endosaccular approach of coiling, the recent paradigm shift of endoluminal parent vessel reconstruction with flow diversion yields durable aneurysm occlusion rates [12-15]. Improvements in robust polyaxial catheter access platforms have facilitated the use of flow diverters, such as the Pipeline embolization device (PED; Medtronic Neurovascular, Irvine, CA, USA), to treat aneurysms in more distal intracranial locations $[16,17]$. The durability of flow diversion makes it a favorable approach for managing aneurysm residuum and recurrences. Recently, Ding and colleagues [18] reported the successful use of PED for the treatment of two recurrent internal carotid artery (ICA) aneurysms that were previously clipped. In this report, we demonstrate the PED treatment of recurrences in previously clipped ACom region aneurysms. This is the first series of its kind describing the success of flow diversion as an alternative management strategy for aneurysm recurrences in this location.

\section{Patients and Methods}

Patient Selection

We retrospectively reviewed a prospective, single-center aneurysm database identifying all patients with recurrent or residual ACom or A1-A2 junction aneurysms after microsurgical clipping. Cases that subsequently underwent endovascular treatment using the PED were identified for analysis.

Data Collection

Data were collected with respect to patient demographics, initial aneurysm status (ruptured/subarachnoid hemorrhage or unruptured), proximal tortuosity, procedural equipment, and technical details. Factors assessed for proximal tortuosity included cervical ICA tortuosity (defined as a 90-degree turn, hairpin turn, or corkscrew loop) and cavernous ICA grade [19]. Data were presented as counts.

Endovascular Procedure

Details regarding the embolization procedure have been previously outlined [13, 20]. Patients were preoperatively treated with daily aspirin $325 \mathrm{mg}$ and clopidogrel $75 \mathrm{mg}$ daily for at least 5 days prior to the treatment. P2Y12 assay for platelet inhibition testing was not routinely performed. Systemic anticoagulation with heparin was given during the embolization. Through an 8-Fr femoral sheath, a triaxial system was used for all procedures consisting of either a 6-Fr 087 Flexor Shuttle guiding sheath (Cook Medical, Bloomington, IN, USA) or an 8-Fr 088 Neuron MAX delivery catheter (Penumbra, Alameda, CA, USA), a 5-Fr distal intracranial catheter, either Navien (Medtronic Neurovascular, Irvine, CA, USA) or Catalyst 5 (Stryker Neurovas- 

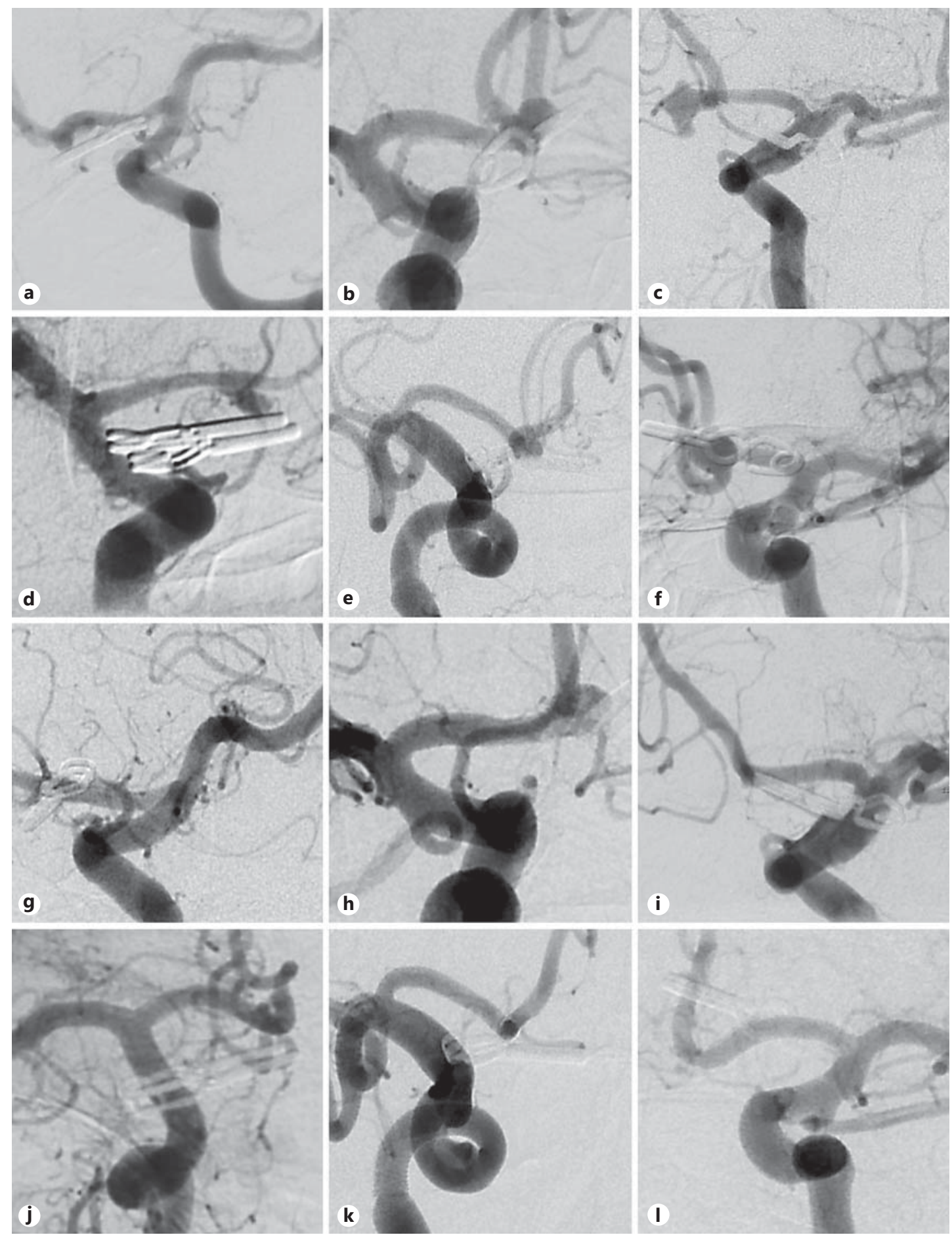

Fig. 1. Pipeline embolization of recurrent post-clipping anterior communicating artery (ACom) region aneurysms. a-f Post-clipping recurrences of the 6 ACom region aneurysms prior to treatment with Pipeline embolization device (PED). g-I Post-PED treatment follow-up angiography demonstrating complete angiographic occlusion in all except case No. 2 (h). 


\section{DOI: $10.1159 / 000460264$ \\ Lin et al.: Rescue Treatment with Pipeline Embolization for Postsurgical Clipping \\ Recurrences of Anterior Communicating Artery Region Aneurysms}

cular, Freemont, CA, USA), and a 027 microcatheter, either Marksman (Medtronic Neurovascular) or VIA27 (Sequent Medical, Aliso Viejo, CA, USA). Parent vessel measurements were made using digital subtraction angiography, and PED deployment occurred under real-time fluoroscopy. Angiography was performed in both an immediate and delayed fashion after deployment to determine adequate PED positioning and parent vessel patency.

\section{Results}

A total of 9 PED neurointerventions were performed for the management of 6 aneurysm recurrences after surgical clipping in the ACom region (Fig. 1). Tables 1 and 2 summarize the details of the cases.

\section{Patient and Aneurysm Characteristics}

Four men and 2 women were treated. Mean patient age was $59.5 \pm 6.9$ years with a range of 50-67 years of age. A total of 6 aneurysms were treated (one patient had bilateral PED placement ("H-pipe") followed by double coverage PED placement on the right side, one patient had an unsuccessful attempt with the Marksman microcatheter and subsequently had a successful PED deployment using the VIA27 microcatheter). Four aneurysms were located on the ACom and 2 were on the A1-A2 junction. Of the 6 aneurysms treated, 4 were previously ruptured. Mean aneurysm size was $5.1 \pm 2.2 \mathrm{~mm}$ with a range of 3-9 $\mathrm{mm}$.

\section{Proximal Vascular Access Characteristics}

Indicators of proximal access complexity and procedural challenges include cervical ICA tortuosity and cavernous ICA grade. Of the 9 cases, 5 (56\%) had significant cervical ICA tortuosity defined as a 90-degree turn, hairpin turn, or corkscrew loop. A spectrum of cavernous ICA tortuosity was encountered: type IA ( $n=1)$, type IB $(n=1)$, type II $(n=3)$, type III $(n=2)$, type IV $(n=2)$. Positions of the distal intracranial catheters used were as follows: proximal cavernous $(n=4)$, distal cavernous $(n=3)$, supraclinoid $(n=2)$.

\section{Procedural Characteristics}

A single PED, deployed from ipsilateral A2 to ipsilateral A1, was used in 6 cases. One case required bilateral PED implantation in a sequential fashion, forming an $\mathrm{H}$-type configuration resulting in endovascular disconnection of the ACom. This same case subsequently required a third PED implantation to double cover the right A1-A2. Another case was an initial failed attempt with the Marksman microcatheter and required corking of the PED. The case was reattempted using the VIA27 microcatheter with successful PED deployment. Balloon postprocessing of the PED was required in 1 case. Mean radiation exposure for the 9 cases was $2,307 \pm 735 \mathrm{mGy}$. Mean fluoroscopy time was $44.1 \pm 12.4 \mathrm{~min}$. The following PED diameters were used in the cases: $3.5 \mathrm{~mm}(n=1), 3.25 \mathrm{~mm}(n=1), 2.75 \mathrm{~mm}(n=3), 2.5 \mathrm{~mm}(n=3)$. No instances of periprocedural complications were encountered.

Follow-Up

All of the aneurysms treated had angiographic follow-up, with 5 of these (83\%) demonstrating complete angiographic occlusion of the aneurysms.

\section{Case Presentations}

Case No. 4

A quinquagenarian presented with loss of consciousness after a witnessed new-onset seizure. CT imaging demonstrated diffuse subarachnoid hemorrhage with interhemispheric 
Table 1. Case summaries

$$
\text { Number/mean (range) Percent/standard deviation }
$$

Demographics

\begin{tabular}{|c|c|c|}
\hline Total patients & 6 & \\
\hline Age & 59.5 years $(50-67)$ & \pm 6.9 \\
\hline Female sex & 2 & $33 \%$ \\
\hline Aneurysms treated & 6 & \\
\hline Size, average & $5.1 \mathrm{~mm}(3-9)$ & \pm 2.2 \\
\hline \multicolumn{3}{|l|}{ Aneurysm location } \\
\hline Anterior communicating artery & 4 & $66 \%$ \\
\hline $\mathrm{A} 1-\mathrm{A} 2$ & 2 & $33 \%$ \\
\hline Prior subarachnoid hemorrhage & 4 & $66 \%$ \\
\hline \multicolumn{3}{|l|}{ Access, catheters, and anatomy } \\
\hline Total cases & 9 & \\
\hline \multicolumn{3}{|l|}{ Distal intracranial catheter } \\
\hline Navien $0.058 ”$ & 7 & $78 \%$ \\
\hline Catalyst 5 0.058” & 2 & $22 \%$ \\
\hline \multicolumn{3}{|l|}{ Microcatheter used } \\
\hline Marksman 0.027" & 6 & $67 \%$ \\
\hline Via $0.027 "$ & 3 & $33 \%$ \\
\hline $\begin{array}{l}\text { Significant cervical internal carotid } \\
\text { artery tortuosity }\end{array}$ & Significant cervical internal carotid & $56 \%$ \\
\hline \multicolumn{3}{|l|}{ Cavernous internal carotid artery grade } \\
\hline IA & 1 & $11 \%$ \\
\hline IB & 1 & $11 \%$ \\
\hline II & 3 & $33 \%$ \\
\hline III & 2 & $22 \%$ \\
\hline IV & 2 & $22 \%$ \\
\hline \multicolumn{3}{|l|}{ Distal intracranial catheter position } \\
\hline Proximal cavernous & 4 & $44 \%$ \\
\hline Distal cavernous & 3 & $33 \%$ \\
\hline Supraclinoid & 2 & $22 \%$ \\
\hline \multicolumn{3}{|l|}{ Case characteristics } \\
\hline Fluoroscopy time & $44.1 \min (30-71)$ & \pm 12.4 \\
\hline Radiation exposure & 2,307 mGy $(1,153-3,487)$ & \pm 735 \\
\hline Adjunct coil deployment & 0 & $0 \%$ \\
\hline Spasm (verapamil) & 1 & $13 \%$ \\
\hline Balloon post-processing & 1 & $13 \%$ \\
\hline Intraoperative rupture & 0 & $0 \%$ \\
\hline Pipeline embolization device cork/removal & 2 & $22 \%$ \\
\hline
\end{tabular}

intracerebral hemorrhage and intraventricular hemorrhage. Cerebral angiography demonstrated $2 \mathrm{~mm}$ anteroinferiorly projecting ACom aneurysm (Fig. 2a). The patient underwent right-sided pterional craniotomy for microsurgical clipping of the aneurysm with complete clip occlusion of the aneurysm neck. Follow-up cerebral angiography at 2 months' time demonstrated regrowth of the ACom aneurysm to $3.7 \mathrm{~mm}$ (Fig. 2b). Given the higher risks of intraprocedural rupture with coiling small aneurysms, PED treatment was elected, with the added advantage of durability with flow diversion. The patient was started on aspirin $325 \mathrm{mg}$ and clopidogrel $75 \mathrm{mg}$ daily 7 days prior to the planned Pipeline embolization treatment of this recurrent aneurysm. A $2.5 \times 14 \mathrm{~mm}$ PED was successfully deployed from the right A2 into 
Lin et al.: Rescue Treatment with Pipeline Embolization for Postsurgical Clipping Recurrences of Anterior Communicating Artery Region Aneurysms

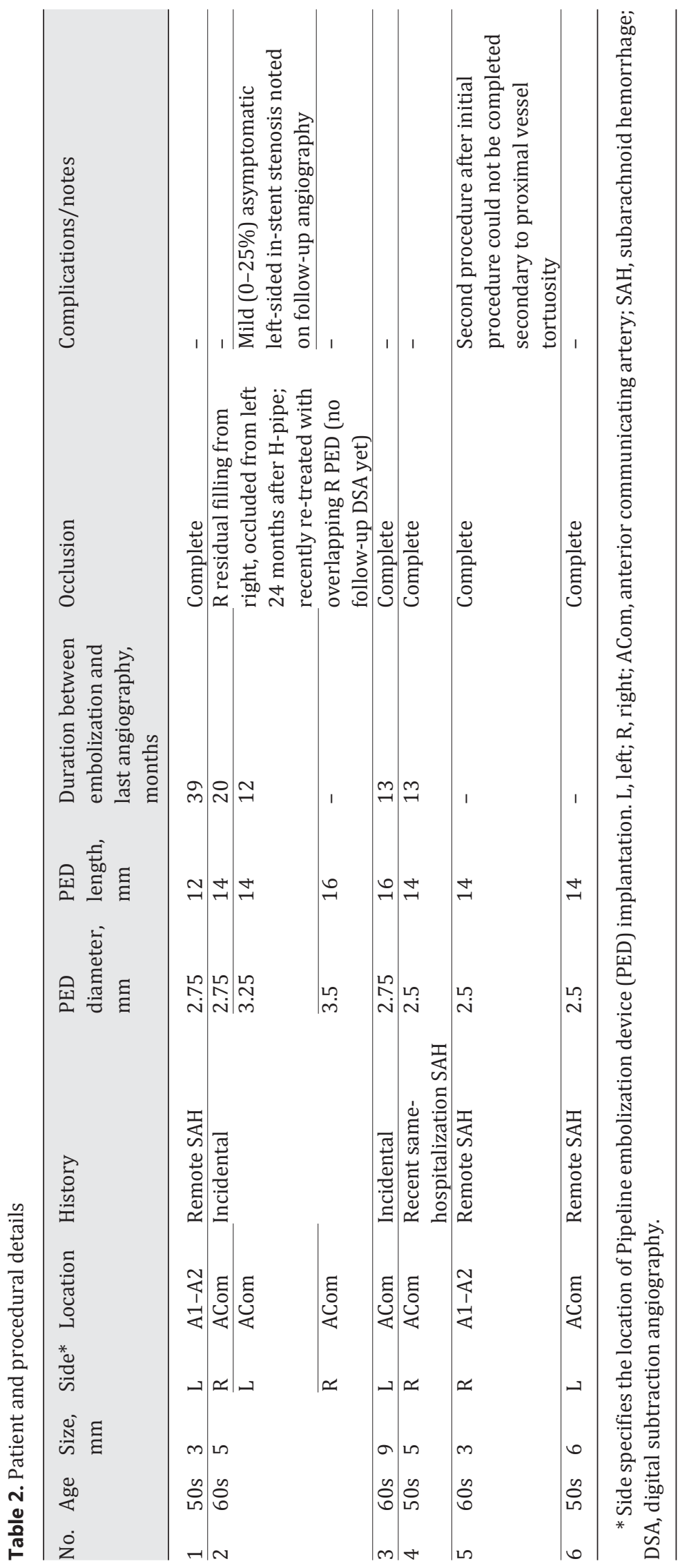


Fig. 2. Pipeline embolization of recurrent post-clipping anterior communicating artery (ACom) aneurysm: case No. 4. a Transorbital oblique digital subtraction angiography (DSA) of the right internal carotid artery (ICA) demonstrating a 2-mm ACom aneurysm (arrowhead) in a patient with grade IV subarachnoid hemorrhage. b Right ICA DSA 2 months following microsurgical clipping demonstrates aneurysmal recurrence (arrow). c Native fluoroscopic image, lateral view, demonstrates Marksman microcatheter (arrow) and Pipeline embolization device (PED; asterisk) deployed from the right A2 to right A1 segments for endoluminal coverage of the aneurysm recurrence. d Right ICA DSA 12 months following PED placement demonstrating that flow remodeling has occurred with successful complete angiographic occlusion of the aneurysm recurrence (arrow).
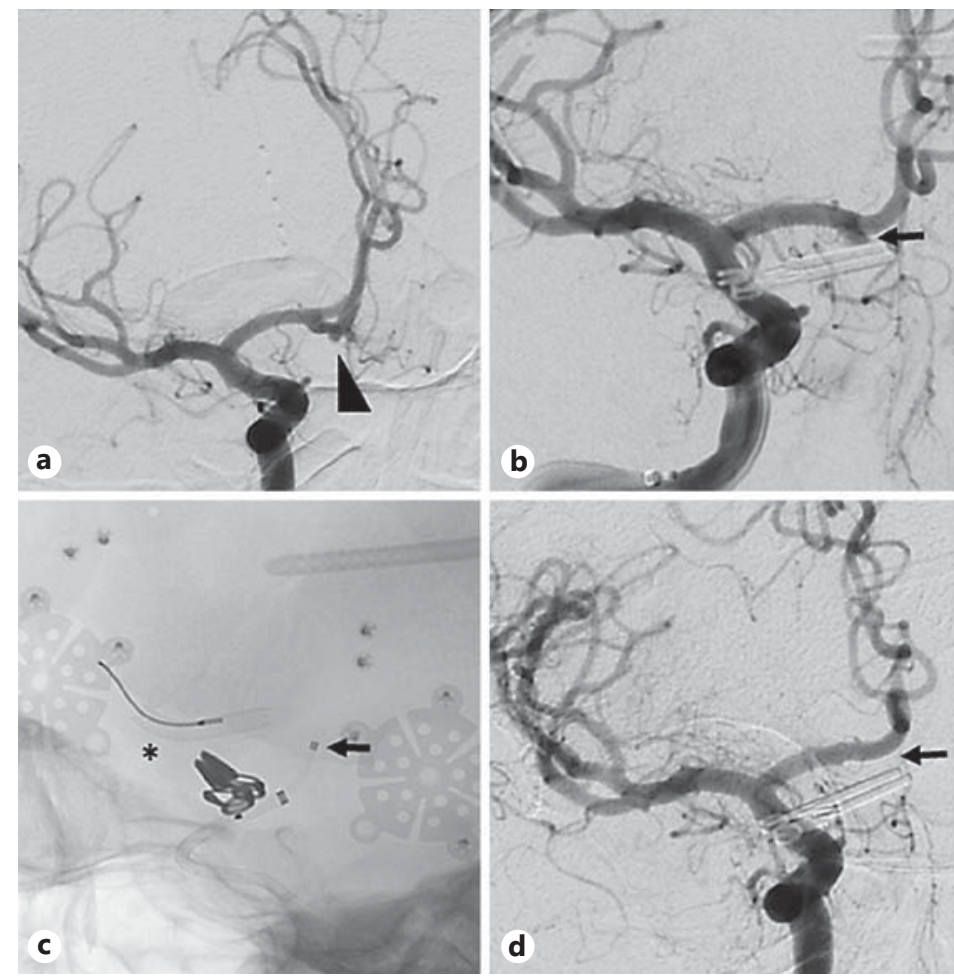

the right A1 without event (Fig. 2c). The patient had an unremarkable postoperative course and was discharged at his neurological baseline. Twelve-month follow-up angiography demonstrated complete aneurysmal occlusion (Fig. 2d).

Case No. 3

A sexagenarian with remote history of subarachnoid hemorrhage from rupture of an ACom region aneurysm presented with episodes of diplopia and transient ischemic attacklike symptoms. Diagnostic cerebral angiography revealed an irregular, left A1-A2 junction aneurysm measuring approximately $9 \times 6 \mathrm{~mm}$ (Fig. 3a, b). He was started on daily aspirin 325 mg and clopidogrel $75 \mathrm{mg} 7$ days prior to the planned PED treatment. He subsequently underwent Pipeline embolization for treatment of the aneurysm recurrence with a $2.75 \times 16$ mm PED (Fig. 3c). Follow-up angiography 12 months later demonstrated complete occlusion of the aneurysm (Fig. 3d).

Case No. 2

A sexagenarian was found incidentally to have a 6-mm right filling ACom aneurysm on MRI during a right middle cerebral artery stroke evaluation (Fig. 4a). After recovering from the stroke, he underwent elective right-sided microsurgical clipping of the aneurysm. Intraoperative angiography had confirmed complete angiographic occlusion at the time. Cerebral angiography at 1 year demonstrated $5 \mathrm{~mm}$ neck recurrence (Fig. $4 \mathrm{~b}, \mathrm{c}$ ). The patient was started on aspirin $325 \mathrm{mg}$ and clopidogrel $75 \mathrm{mg}$ daily 7 days prior to the planned Pipeline embolization treatment of this recurrent aneurysm. A $2.75 \times 14 \mathrm{~mm}$ PED was successfully deployed from the right A2 into the right $A 1$ without event. Cerebral angiography at 6 months 
Fig. 3. Pipeline embolization of recurrent anterior communicating artery (ACom) aneurysm with remote history of microsurgical clipping: case No. 3. a, b Pre-embolization left internal carotid artery (ICA) digital subtraction angiography (DSA) runs demonstrating an irregularly shaped, medially projecting ACom aneurysmal recurrence (arrowhead). c Native fluoroscopic image, lateral view, demonstrates Pipeline embolization device deployment across the neck of the aneurysm recurrence. The device (asterisk) is deployed from ipsilateral left A2 to left A1 segments in a distal to proximal fashion using a Marksman microcatheter (arrow). d Left ICA DSA demonstrating complete angiographic occlusion of the aneurysmal recurrence (arrow) at 12 months' follow-up.

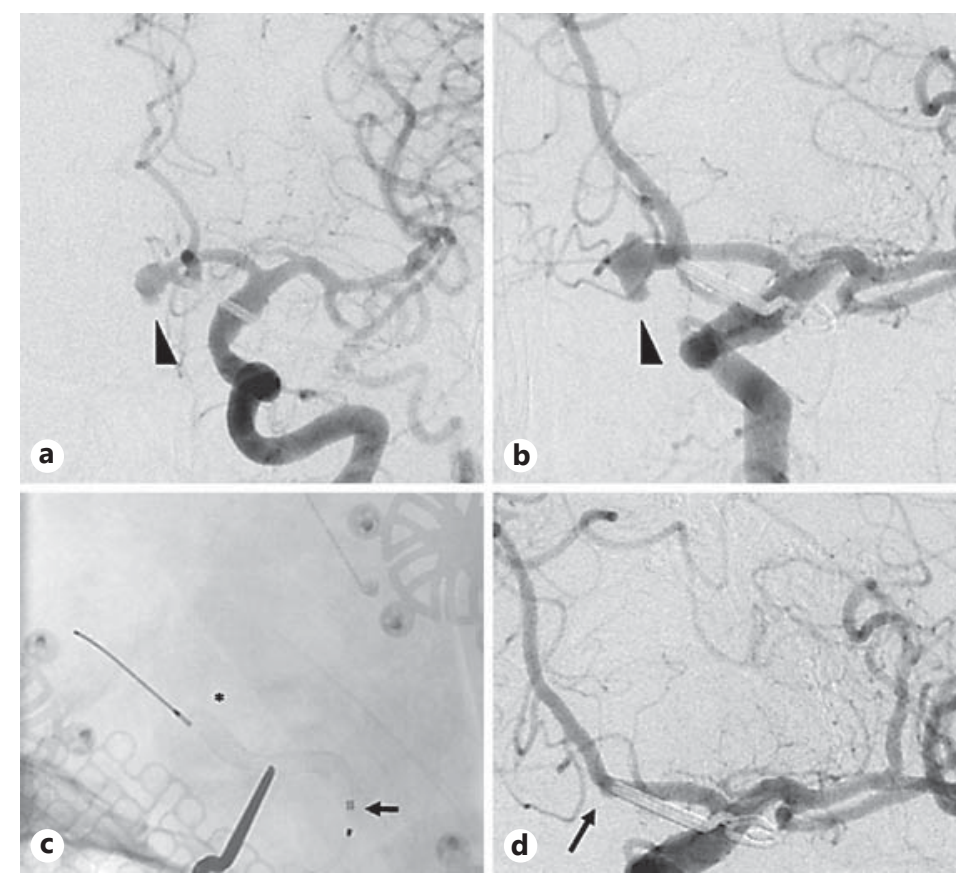

demonstrated interval filling of the aneurysm from the left anterior circulation and persistent filling from the right side (Fig. 4d, e). A second PED, $3.25 \times 14 \mathrm{~mm}$, was placed from left A2-A1 without event. Follow-up angiography at 2 years demonstrated no filling from the left side but persistent slow filling from the right (Fig. 4f, g). At this time, the patient was only on daily aspirin $81 \mathrm{mg}$. The patient subsequently was restarted on aspirin $325 \mathrm{mg}$ and clopidogrel 75 mg daily for 7 days prior to additional PED treatment. A $3.5 \times 16 \mathrm{~mm}$ PED was successfully deployed within the first PED on the right side. Final procedural control angiography demonstrated mild reduction in the aneurysm filling (Fig. 4h, i). Follow-up angiography is pending.

\section{Discussion}

Management of recurrent or remnant aneurysms can be particularly challenging from both a microsurgical and a traditional endovascular perspective. Reoperative management of aneurysm remnants involves risk factors such as scarring, stroke, seizures, wound healing complications, and postoperative pain [21]. In comparison, traditional endosaccular treatment modalities for recurrent or remnant aneurysms may result in incomplete neck obliteration or coil herniation into the parent vessel, particularly for wide-neck aneurysms. Balloon or stent assistance can be used to improve aneurysm neck coverage with coil embolization; however, these techniques can be associated with an increased risk of thromboembolism, dissection, and intraprocedural rupture [11,22, 23].

Flow diversion may be an ideal means by which recurrent aneurysms at the ACom region can be treated. Advantages include a non-intrasaccular approach for high-risk remnants or recurrences that are wide neck and/or anatomically complex. Additionally, flow diversion allows for parent vessel reconstruction through progressive aneurysm sac thrombosis with neointimal proliferation at the aneurysm neck, resulting in complete occlusion of these 

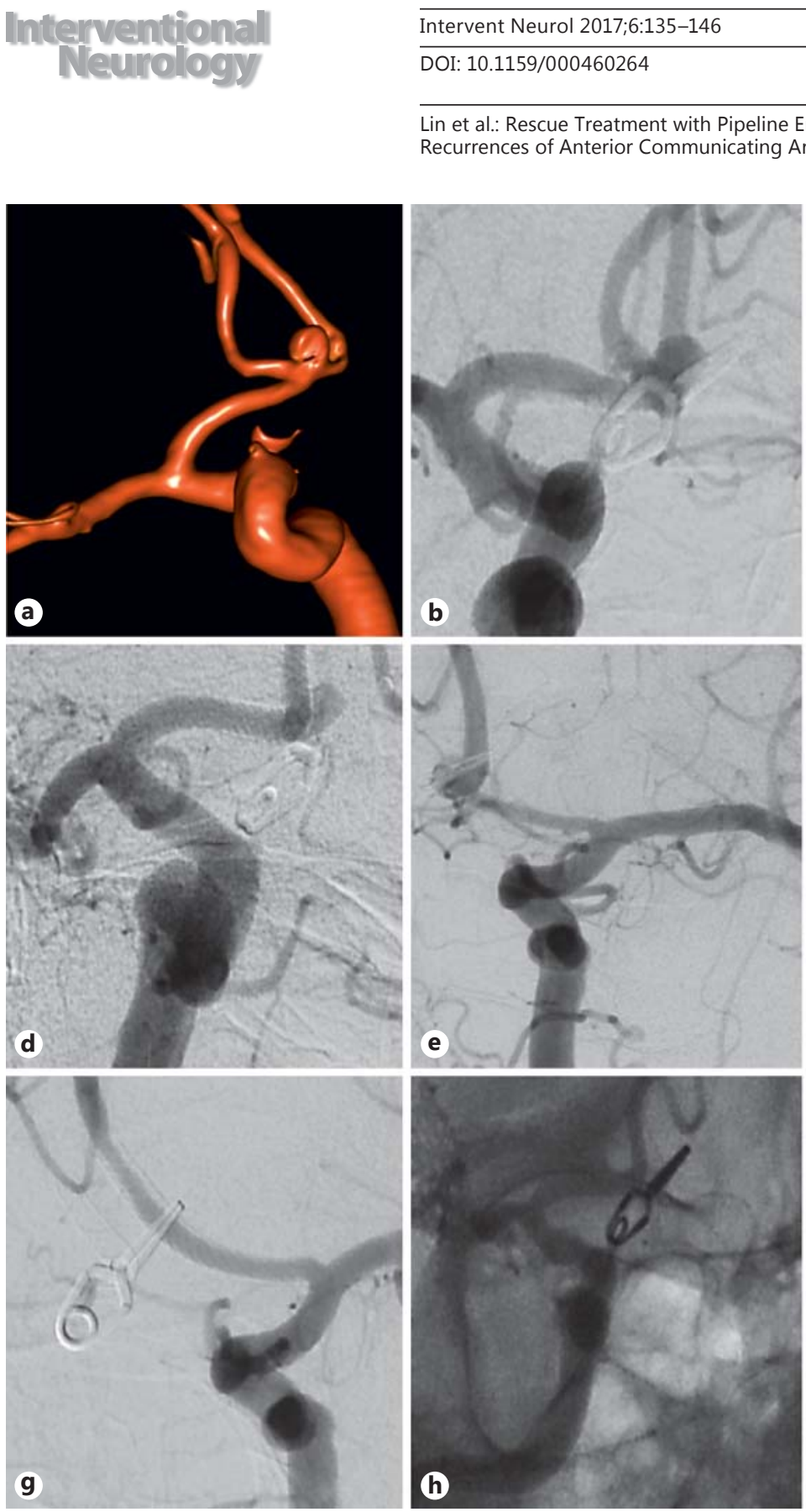

Intervent Neurol 2017;6:135-146

DOI: $10.1159 / 000460264$
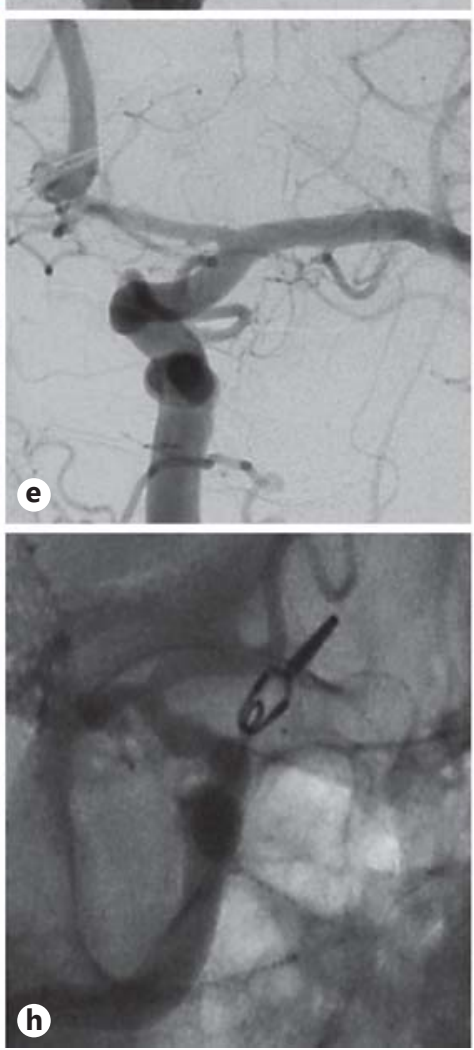

Lin et al.: Rescue Treatment with Pipeline Embolization for Postsurgical Clipping Recurrences of Anterior Communicating Artery Region Aneurysms
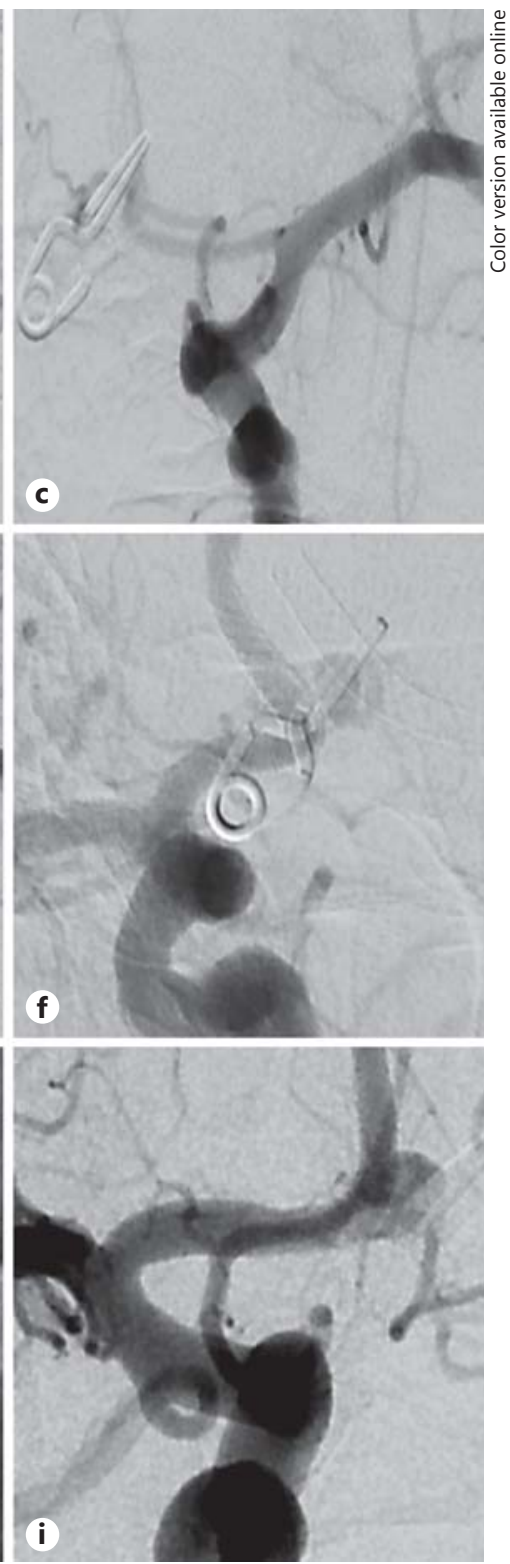

Fig. 4. Pipeline embolization in $\mathrm{H}$ configuration of recurrent post-clipping anterior communicating artery (ACom) aneurysm: case No. 2. a Three-dimensional reconstruction image of right filling ACom aneurysm. b Right internal carotid artery (ICA) digital subtraction angiography (DSA) 1 year following microsurgical clipping demonstrated $5 \mathrm{~mm}$ neck recurrence. c Left ICA DSA 1 year following microsurgical clipping demonstrates aneurysm fills only from the right ICA injection. d Right ICA DSA 6 months following Pipeline embolization device (PED) implantation from the right A2 into right A1 demonstrated persistent aneurysm filling. e Left ICA DSA 6 months following PED implantation from the right A2 into the right A1 demonstrated interval flow alteration with filling of the aneurysm now also from the left anterior circulation. $\mathbf{f}$ Right ICA DSA 2 years following PED implantation from the left A2 into left A1 demonstrated continued aneurysm filling. $g$ Left ICA DSA 2 years following PED implantation from the left A2 into left A1 demonstrated no further filling of the aneurysm from the left anterior circulation. $\mathbf{h}$ Native fluoroscopic image (anterior-posterior view) of third PED telescoped within the first PED on the right side. i Right ICA DSA final control angiography during third PED implantation demonstrates mild decreased contrast filling of the aneurysm. 
Lin et al.: Rescue Treatment with Pipeline Embolization for Postsurgical Clipping

Recurrences of Anterior Communicating Artery Region Aneurysms

lesions [12]. There is already growing interest in using flow diversion techniques to treat anterior circulation aneurysms beyond the ICA [24-27]. Improvements in robust polyaxial catheter access platforms have facilitated the use of flow diverters, such as the PED, in the cerebrovasculature as distal as the A2-A3 junction of the anterior cerebral artery or the M2 segment of the middle cerebral artery $[16,17]$.

Experience with flow diversion technique for treatment of ACom region aneurysms remains sparse in the literature and is often described in combination with experiences in bifurcation aneurysms. For example, Saleme and colleagues [26] reported on the PED treatment of 37 bifurcation aneurysms; however, only 9 of these aneurysms were located in the ACom region. Although 6 of these 9 ACom region aneurysms were recurrences, no information was provided on whether these were recurrences after coiling or clipping. Similarly, Gawlitza and colleagues [24] reported a series of 18 bifurcation aneurysms treated with a variety of flow diverters, with only 5 of these aneurysms located in the ACom region and only 3 of these 5 aneurysms were recurrences from incomplete coilings. Both the reports published by Saleme et al. [26] and Gawlitza et al. [24] were limited in procedural data and were rather dedicated to evaluating the outcomes of covered cortical branches and perforating arteries. These studies concluded the feasibility of using flow diversion for treatment of bifurcation aneurysms. The heterogeneity in the literature on PED use in ACom region aneurysms limits adequate direct comparison of results reported in these studies. Our current series adds to the literature by providing a dedicated publication with data specific for ACom region aneurysms treated with PED.

In this study, we demonstrate the first reported use of PED treatment in the management of recurrent ACom region aneurysms after surgical clipping. Six aneurysms were treated without any periprocedural complications related to PED, such as intraprocedural thrombosis or intraparenchymal hemorrhages. Our experience suggests that endoluminal reconstruction of these aneurysms via PED flow diversion can be safe and efficacious. In all the cases, small-diameter PED sizes were used, which minimizes concerns for device opening failures associated with larger-diameter devices [28]. Furthermore, these small PED sizes are still relatively oversized in the anterior cerebral artery segments implanted, theoretically minimizing the effect of covering perforators. These factors likely lend to the feasibility of a PED treatment approach for managing these previously clipped recurrent ACom region aneurysms. Additionally, we observed complete angiographic occlusion in 5 of the 6 (83\%) aneurysms on follow-up angiography. There was only one case of mild $(<25 \%)$ in-stent stenosis that was clinically asymptomatic. The one aneurysm with persistent filling required bilateral PED implantation in an "H-type" configuration for endovascular disconnection of the ACom segment followed by subsequent double coverage of the right A1-A2 with an additional PED implantation after continued filling on additional follow-up. Although we have demonstrated successful PED implantation and aneurysm occlusion for recurrent ACom region aneurysms after surgical clipping, long-term follow-up with a larger cohort of patients will be needed to draw further conclusions.

\section{Conclusion}

Flow diversion via PED can be considered as a treatment alternative for recurrent ACom region aneurysms after surgical clipping. This method of endoluminal parent vessel reconstruction avoids risks of potential intraprocedural aneurysm rupture associated with endosaccular coiling and risks of reoperative open microsurgical clipping. The smaller PED sizes used for these cases lend to the feasibility of this approach. Robust polyaxial catheter access platforms also facilitate the use of flow diverters in these distal intracranial locations. 
Lin et al.: Rescue Treatment with Pipeline Embolization for Postsurgical Clipping

Recurrences of Anterior Communicating Artery Region Aneurysms

\section{Statement of Ethics}

All individual identifying information, including age and sex, have been meticulously anonymized to achieve appropriate anonymity in this paper.

\section{Disclosure Statement}

A.L.C. is a proctor for the Woven Endobridge (WEB) device (Sequent Medical, Aliso Viejo, CA, USA), a proctor for the Surpass device (Stryker Neurovascular, Fremont, CA, USA) and a consultant for Stryker Neurovascular, a proctor for the Pipeline Embolization Device (Medtronic Neurovascular, Irvine, CA, USA) and a consultant for Covidien, and a proctor for the FRED device (Microvention, Tustin, CA, USA) and a consultant for Microvention. G.P.C. is a consultant for Covidien and Microvention. L.-M.L. is a proctor for the Pipeline Embolization Device (Medtronic Neurovascular). The other authors have no conflict of interest. No author received financial support in conjunction with the generation of this submission. This research received no specific grant from any funding agency in the public, commercial, or not-for-profit sectors.

\section{Authors' Contributions}

L.-M.L. drafted the manuscript and critically revised the manuscript for important intellectual content. R.R.I., M.T.B., and T.M. assisted with the data collection and analysis. G.P.C. assisted in critically revising the manuscript. A.L.C. conceived of the manuscript and critically reviewed the important intellectual content. All authors read and approved the final manuscript.

\section{References}

1 Steklacova A, Bradac 0, Charvat F, et al: "Clip first" policy in management of intracranial MCA aneurysms: single-centre experience with a systematic review of literature. Acta Neurochir (Wien) 2016;158:533-546; discussion 546.

2 Kozba-Gosztyla M, Czapiga B, Jarmundowicz W: Aneurismal subarachnoid hemorrhage: who remains for surgical treatment in the post-ISAT era? Arch Med Sci 2015;11:536-543.

3 Feuerberg I, Lindquist C, Lindqvist M, et al: Natural history of postoperative aneurysm rests. J Neurosurg 1987;66:30-34.

4 Sindou M, Acevedo JC, Turjman F: Aneurysmal remnants after microsurgical clipping: classification and results from a prospective angiographic study (in a consecutive series of 305 operated intracranial aneurysms). Acta Neurochir (Wien) 1998;140:1153-1159.

5 Macdonald RL, Wallace MC, Kestle JR: Role of angiography following aneurysm surgery. J Neurosurg 1993;79: 826-832.

6 Cekirge HS, Islak C, Firat MM, et al: Endovascular coil embolization of residual or recurrent aneurysms after surgical clipping. Acta Radiol 2000;41:111-115.

7 Rabinstein AA, Nichols DA: Endovascular coil embolization of cerebral aneurysm remnants after incomplete surgical obliteration. Stroke 2002;33:1809-1815.

8 Mangiafico S, Cellerini M, Villa G, et al: Endovascular coiling of aneurysm remnants after clipping in patients with follow-up. A single center experience. Interv Neuroradiol 2005;11:41-48.

9 Vinuela F, Duckwiler G, Mawad M: Guglielmi detachable coil embolization of acute intracranial aneurysm: perioperative anatomical and clinical outcome in 403 patients. J Neurosurg 1997;86:475-482.

10 Raymond J, Guilbert F, Weill A, et al: Long-term angiographic recurrences after selective endovascular treatment of aneurysms with detachable coils. Stroke 2003;34:1398-1403.

11 Nishido H, Piotin M, Bartolini B, et al: Analysis of complications and recurrences of aneurysm coiling with special emphasis on the stent-assisted technique. AJNR Am J Neuroradiol 2014;35:339-344.

12 Becske T, Kallmes DF, Saatci I, et al: Pipeline for uncoilable or failed aneurysms: results from a multicenter clinical trial. Radiology 2013;267:858-868.

13 Colby GP, Lin LM, Gomez JF, et al: Immediate procedural outcomes in 35 consecutive pipeline embolization cases: a single-center, single-user experience. J Neurointerv Surg 2013;5:237-246.

14 Lin LM, Colby GP, Kim JE, et al: Immediate and follow-up results for 44 consecutive cases of small $(<10 \mathrm{~mm})$ internal carotid artery aneurysms treated with the pipeline embolization device. Surg Neurol Int 2013;4:114.

15 Chalouhi N, Zanaty M, Whiting A, et al: Safety and efficacy of the Pipeline Embolization Device in 100 small intracranial aneurysms. J Neurosurg 2015;122:1498-1502. 
Lin et al.: Rescue Treatment with Pipeline Embolization for Postsurgical Clipping

Recurrences of Anterior Communicating Artery Region Aneurysms

16 Lin LM, Colby GP, Huang J, et al: Ultra-distal large-bore intracranial access using the hyperflexible Navien distal intracranial catheter for the treatment of cerebrovascular pathologies: a technical note. J Neurointerv Surg 2014;6:301-307.

17 Lin LM, Iyer RR, Jiang B, Colby GP, Huang J, Tamargo RJ, Coon AL: Pentaxial access platform for ultra-distal intracranial delivery of a large-bore hyperflexible DIC (distal intracranial catheter): a technical note. Interdiscip Neurosurg 2016;6:29-34.

18 Ding D, Starke RM, Evans AJ, et al: Endovascular treatment of recurrent intracranial aneurysms following previous microsurgical clipping with the Pipeline Embolization Device. J Clin Neurosci 2014;21:1241-1244.

19 Lin LM, Colby GP, Jiang B, et al: Classification of cavernous internal carotid artery tortuosity: a predictor of procedural complexity in Pipeline embolization. J Neurointerv Surg 2015;7:628-633.

20 Colby GP, Lin LM, Caplan JM, et al: Immediate procedural outcomes in 44 consecutive Pipeline Flex cases: the first North American single-center series. J Neurointerv Surg 2016;8:702-709.

21 Drake CG, Friedman AH, Peerless SJ: Failed aneurysm surgery. Reoperation in 115 cases. J Neurosurg 1984; 61:848-856.

22 Levy DI, Ku A: Balloon-assisted coil placement in wide-necked aneurysms. Technical note. J Neurosurg 1997; 86:724-727.

23 Moon K, Albuquerque FC, Ducruet AF, et al: Balloon remodeling of complex anterior communicating artery aneurysms: technical considerations and complications. J Neurointerv Surg 2015;7:418-424.

24 Gawlitza M, Januel AC, Tall P, et al: Flow diversion treatment of complex bifurcation aneurysms beyond the circle of Willis: a single-center series with special emphasis on covered cortical branches and perforating arteries. J Neurointerv Surg 2016;8:481-487.

25 Pistocchi S, Blanc R, Bartolini B, et al: Flow diverters at and beyond the level of the circle of Willis for the treatment of intracranial aneurysms. Stroke 2012;43:1032-1038.

26 Saleme S, Iosif C, Ponomarjova S, et al: Flow-diverting stents for intracranial bifurcation aneurysm treatment. Neurosurgery 2014;75:623-631; quiz 631.

27 Yavuz K, Geyik S, Saatci I, et al: Endovascular treatment of middle cerebral artery aneurysms with flow modification with the use of the pipeline embolization device. AJNR Am J Neuroradiol 2014;35:529-535.

28 Lin LM, Colby GP, Jiang B, et al: Intra-DIC (distal intracranial catheter) deployment of the Pipeline embolization device: a novel rescue strategy for failed device expansion. J Neurointerv Surg 2016;8:840-846. 